\title{
The Scope and Applications of Artificial Intelligence in the Medical Sector
}

\author{
Madhuri Kumar and John Alen \\ Bioengineering, The Grainger College of Engineering, University of Illinois, IL 61801, USA \\ Kumar99881@hotmail.com
}

\begin{abstract}
Article Info
Journal of Biomedical and Sustainable Healthcare Applications (http://anapub.co.ke/journals/jbsha/jbsha.html) Doi: https://doi.org/10.53759/0088/JBSHA202101003
\end{abstract}

Received 18 August 2020; Revised form 22 September 2020; Accepted 22 October 2020.

Available online 05 January 2021.

(C2021 Published by AnaPub Publications.

\begin{abstract}
The terminology Artificial Intelligence (AI) describes the application computing systems and technology to effectively simulate smart actions and smart thinking compared to the human mind. The concept of AI was introduced as the engineering and science of making smart machines that can operate without the engagement of humans using Machine Learning (ML). This research provides a wider scope of the concept of $\mathrm{AI}$ in the medical field, handling the various concepts and terms associated with the concept, including the present and future implementation of the concept. The major research materials applied are Google and PubMed searches, which were conducted using the "Artificial Intelligence" as the basic keyword. More references were retrieved by crossreferencing major publications. The advancements in AI technology in recent times and the present application of medicine have been analyzed critically. This paper ends with an assumption that AI focuses on implementing changes in the medical practices in previously unidentified ways. However, many of the application are still in the initial stages and require exploration and development. In addition, clinical experts have to comprehend and adapt with development for effective delivery of medical services.
\end{abstract}

Keywords - Artificial Intelligence (AI), Machine Learning (ML), AI Algorithms.

\section{INTRODUCTION}

Several clinical issues are ideal for AI technologies, because to advances in computing power and large quantities of data produced in medical systems. The following are two recent examples of reliable and medically relevant heuristics that may help both physicians and patients by simplifying diagnosis. One of the many known instances of AI Algorithms that surpass physicians in picture classification techniques is the first of such techniques. Scientists at Seoul Health Center and College of Medicine created the DLAD (Machine Learning based Automated Diagnosis) AI system to scan plain radiography and identify aberrant cell development, such as possible malignancies [1], in the autumn of 2018 (see Fig 1). On the same pictures, the application's results are compared to that of various physicians' detecting skills, and it surpassed $17 / 18$ physicians.
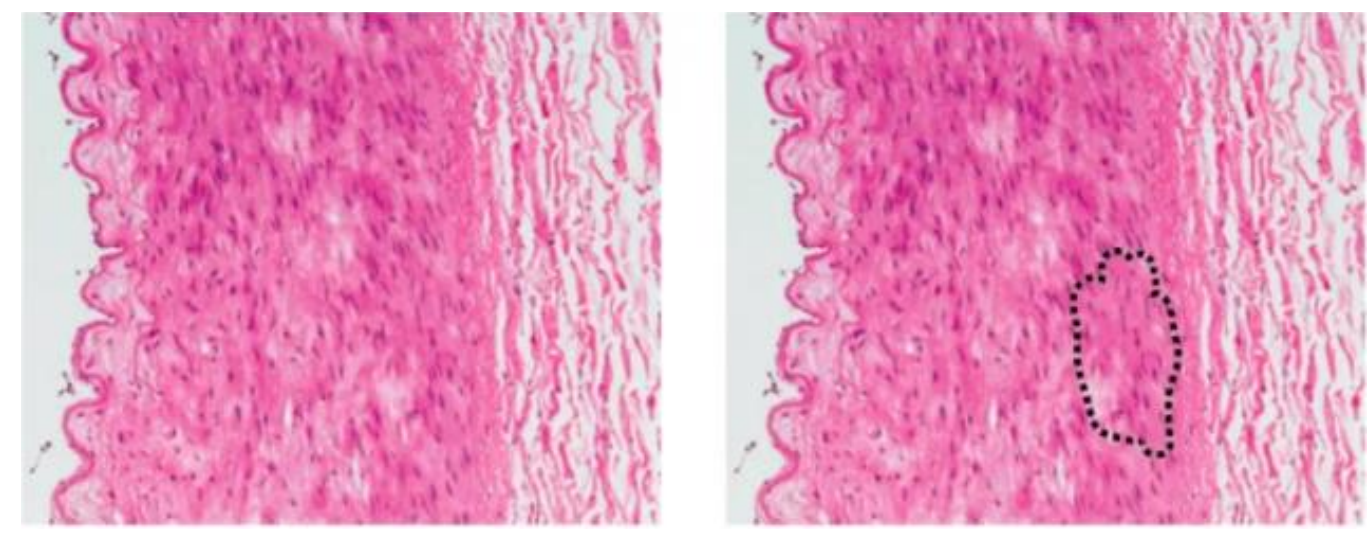

Fig 1. AI algorithmic application in medicine.

In the leftmost column, you can see the image that was used to train AI applications. According to Automated Processes, the right column illustrates a region of potentially harmful cells that a doctor should study further.

The second of such programs was developed by Google Ml Medical scientists in the autumn of 2018, which produced LYNA (Lymph Node Assistant), a training system that evaluated histopathology presentations colored biopsy to detect breast carcinoma tumors in lymphadenopathy biopsy [2]. Although this is not the first time Machine learning is often used to assess histopathological, it's important to mention that this approach was able to determine potentially dangerous spots 
in biopsy specimens that were previously undetected to the human eye. On several databases, LYNA was found to correctly classify a specimen as cancerous or premalignant 99 percent of the time. When given to clinicians to use in conjunction with their routine assessment of stained clinical specimens, LYNA also reduced the average slide inspection time by half. Other microscopy technologies have recently shown a comparable capacity to improve physician reliability. In the near term, physicians may utilize these AI Algorithms to help them double-check diagnostics and analyze patient data more quickly without compromising accuracy. In the long run, however, congress programs may be able to operate autonomously in clinics, enabling physicians to concentrate on situations that machines cannot handle. Both DLAD and LYNA are excellent illustrations of programs that help clinicians classify healthy and sick samples by highlighting key characteristics of pictures that should be investigated further. What is stopping AI Algorithms from being utilized in clinical settings? These studies show the potential benefits of methods in healthcare; what is stopping AI Algorithms from being employed in healthcare situations?

In healthcare, strategies have proven to offer many potential advantages for both physicians and patients. Monitoring these systems, on the other hand, is a tough job. Although the US Food and Drug Administration (FDA) has authorized certain assistive technologies, there are presently no uniform clearance criteria [3]. Furthermore, the individuals who create techniques for use in clinics aren't usually the same physicians who treat patients. As a result, computationalists may have to understand further about healthcare, while physicians might have to understand much more about activities for which a certain algorithm is or is not well suited. While AI can assist with diagnostics and basic clinical duties, it's difficult to envision automated brain operations, where physicians must occasionally alter their strategy on the run once they look inside the patient. The potential of AI in healthcare presently exceed the power of AI for acute management in this and other ways. Clarified FDA guidelines, on the other hand, may assist define algorithm criteria and lead to an increase in clinically used techniques.

Additionally, the FDA has stringent clinical trial approval requirements, demanding great openness in scientific procedures. To go from data input to the end output, many techniques depend on very complex, difficult-to-understand mathematics, which is often referred to as a "data recorder." Is it possible that the FDA's failure to 'untangle the data recorder' and explain the internal workings of AI Algorithms would affect the probability of an AI-based study being approved? Researchers, businesses, and innovators are typically wary of disclosing their unique techniques to the public for fear of losing money if their ideas are stolen and enhanced by others. The uncertainty around algorithm details may be reduced if patent rules alter from their present form, where a program is theoretically only protected if it is part of a virtual computer. In any case, greater openness in the near term is essential to ensure that patient data is not mistreated or incorrectly categorized, and to assess if an algorithm will be accurate enough in the clinic. In addition to the challenges of FDA clearance, AI Algorithms may have trouble gaining patient confidence and acceptance.

Patients may be unwilling to allow AI Algorithms be used to assist them with their medical requirements if those authorizing them for clinical usage do not have a good grasp of how they operate. Would patients prefer to be mistreated by a person or a program if the AI Algorithms consistently beat doctors if forced to choose? Many people struggle to answer this question, but it essentially comes down to having faith in a system's decision-making. Effective decision is dependent on the normality of the model utilized as inputs, which is critical for proper operation. With erroneous data, AI Algorithms may provide erroneous results [4]. It's conceivable that the people who create AI Algorithms don't realize the information they feed it is false until it's too late, and their algorithm has resulted in medical negligence. This mistake may be avoided if both doctors and programmers are well-versed in the data and techniques required to properly utilize data in the algorithm. It will be less probable for a program to learn to make erroneous decisions if connections are established between doctors who understand the details of the medical data as well as the computationalists who create the algorithm.

Practitioners must know the scope of techniques, and developers must comprehend medical evidence, in order to create AI Algorithms that can be used in the clinic. It may be essential for businesses to reveal the inner workings of their programs so that a larger audience may scrutinize the techniques and point out potential sources of mistake that might have an effect on patient care. We seem to be a long way from AI Algorithms functioning independently in clinics, particularly given the absence of a clear route to clinical clearance. Defining the qualities that a methodology must possess in order to be deemed satisfactorily precise for use in a clinic, as well as address possible inaccuracies in the algorithm's decisionmaking and being clear as to where a computation excels or where it continues to fail, could pave the way for social approval of methodologies to take over certain tasks from doctors. These obstacles, on the other hand, are worth attempting to surmount in order to improve the precision and effectiveness of therapeutic procedures for a wide range of illnesses. The section below presents a critical review of the relevant literatures regarding the aspect of AI in medicine. Section III focuses on the materials and methods used for the research. Section IV evaluates the results. Section V presents a discussion of the results while Section VI concludes the research.

\section{LITERATURE REVIEW}

A. Hadioui, N. El Faddouli, Y. Benjelloun Touimi and S. Bennani in [5] argue that the impressive capacity of machine learning to absorb massive quantities of data, make logical sense of pictures, and recognize trends that even the most trained human eye misses has sparked optimism that the technologies could revolutionize medicine. Professionals in computer programming, health, politics, economics, philosophy, and other fields will need to work together to maximise the possibilities of this possibility. The Institute for Artificial Intelligence in Medicine and Imaging (AIMI) webcast workshop on August 5 focused on this multidisciplinary methodology. The event examined new clinical artificial intelligence technology with an emphasis on technological breakthroughs, data integrity, legislation, and legislation, and included global leaders in computer engineering, healthcare, trade, and administration. Matthew Lungren, adjunct 
professor of radiography at Stanford and AIMI founder, and Serena Yeung, adjunct professor of genomic data bioinformatics and computational science at Stanford and AIMI senior director, organized the conference [6]. The day's talks were centered on the concept of "correlational research," which refers to the formation of a new intellectual pursuit when professionals from several disciplines engage in an open debate to create a new subject.

Scripps Study's noted cardiology, scientist, and editor A. Esteva and E. Topol in [7] identifies three aspects wherein AI has the most promise to revolutionize healthcare. The first is in minimizing healthcare errors related to misinterpretation, he said, citing a notable NYU research that says that human pathophysiology coupled with Machine learning was good at spotting forms of cancer than either alone, especially in reducing false-negative mammography, which cause many female to wait longer for care. "This is critical information," Topol said. "AI has the ability to perceive things that the human sight cannot." Second, a plethora of new programs will be available to assist people in self-managing their health throughout their lives, such as mobile applications that detect skin malignancies. Topol predicts that this will be the era of the "medical selfie" - snap a photo, receive a diagnosis. Lastly, AI has the potential to "humanize health care" by bringing the doctor closer to the patients. We can reduce or eliminate the tedium of data input, which causes doctor weariness and takes away valuable time with patients, he added. He went on to say that AI's most significant contribution to healthcare will be restoring humanity to the field.

Simply developing medical AI products is not enough. It's all about getting those goods in front of consumers. "The greatest thing in the world is useless if no one can use it," Lily Peng, director of product at Google Neuroscience AI Research Team, said. She cited a recent program her team developed to detect diabetic eye illness as an example of a program that might have achieved consistent outcomes with such a shorter, higher-quality database, possibly assisting in the faster development of a useful product. We need to make these goods more accessible to the general public [8].

D. Nelson in [9] looked at how systems learned on private or inadequate information usually fail beyond those cozy boundaries - in other words, they don't adapt. He cited American-trained Artificial intelligence systems for lung illnesses that do not include TB in their labelling as an instance. Because tuberculosis is a well-known issue in the poor world but not so much in the United States, there are no tuberculosis scans in the training sample. According to him, true egalitarianism necessitates AI working everywhere for everyone. Simply include TB pictures in American training datasets might assist to generalize - and therefore democratising AI in other areas of the globe.

To treat malignancy, TB, Dengue, and even COVID-19, authors in [10] explained how democratize in his native nation of Brazil involves utilizing AI to deliver or speed up treatment when experts and facilities are in limited supply. He pointed out that an AI model for diagnosing Zika from medical pictures provides a useful tool to areas of Brazil — a nation nearly the population of the United States - where access to excellent medical care is limited. The importance of the regulator should not be underestimated by AI, according to the $\mathrm{u}$ head of health policy at the US Food and Drug Administration (FDA). "Regulation is a team effort," he said. "We're worried about privacy, but we would also like to see excellent technology reach the clinics," says one regulator.

P. Boddington, P. Millican and M. Wooldridge in [11] were a pioneer in the development of today's machines and artificial intelligence. The "Completely turning test" was founded on the idea that a computer's intelligence is defined by its ability to perform at a human level in cognition-related activities. Paraphrase that has been formalized AI sparked a surge of interest in the 1980s and 1990s. Fuzzy inference networks, Bayes network, convolutional neural networks, and composite cognitive technologies are among the artificial intelligence methods utilized in health care. When compared to other industries, healthcare systems received the most funding in AI research in 2016. In medicine, artificial intelligence may be divided into two types: physical and virtual.

The virtual component includes anything from electronic health records to machine - learning therapy decision assistance. The physical section covers robotic surgery assistants, intelligent prosthetics for disabled individuals, and geriatric care. The foundation of evidence-based healthcare is the development of connections and trends from a preexisting collection of data in order to create clinical connections and discoveries. To demonstrate these trends and connections in the past, we utilized statistical techniques. Control charts and the databases strategy are two methods for teaching computers how to diagnose a patient.

The flowchart-based method entails adapting the procedure of history-taking, which entails a physician conducting questions and then integrating the symptoms given to arrive at a likely diagnosis. Given the broad variety of ailments and pathological conditions seen in regular medical practice, this necessitates putting a significant quantity of information into machine-based cloud platforms. Because computers are unable to watch and collect signals that can only be noticed by a physician during a patient visit, the results of this method are restricted. The database method, on the other hand, is based on the concept of computational modeling or analytical thinking, which entails training a computer to identify specific groupings of symptoms or diagnostic and therapeutic pictures using repeated AI Algorithms. Google's simulated brain program, which began in 2012, is an illustration of this technique. Based on 10,000,000 Youtube clips, this system taught itself to identify cats, with efficiency increasing as more pictures were reviewed. It could correctly identify a picture of a kitten with $75 \%$ reliability after just 3 days of training. The materials and methods are evaluated in Section III.

\section{MATERIALS AND METHODS}

The main phrase "artificial intelligence" was searched in PubMed and Google. Cross-referencing the main publications yielded additional citations. An overview of several AI-based technologies that are presently in use or in progress is provided. This article serves as a guide to comprehending the core of artificial intelligence in medicine as well as the details of its governance. Dialogical, analytical, empirical, structural, and holistic techniques are used. 


\section{RESULTS}

Many applications of AI are already in use in the healthcare profession, varying from digital appointment booking, online verification in major hospitals, digitalisation of medical information, reminder demands for follow-up consultations and vaccination dates for pregnant women and children to appropriate dosage methodologies and negative affect cautions when allowed to prescribe multi - drug variations. The wide uses of AI in healthcare are shown in the chart Fig 2.

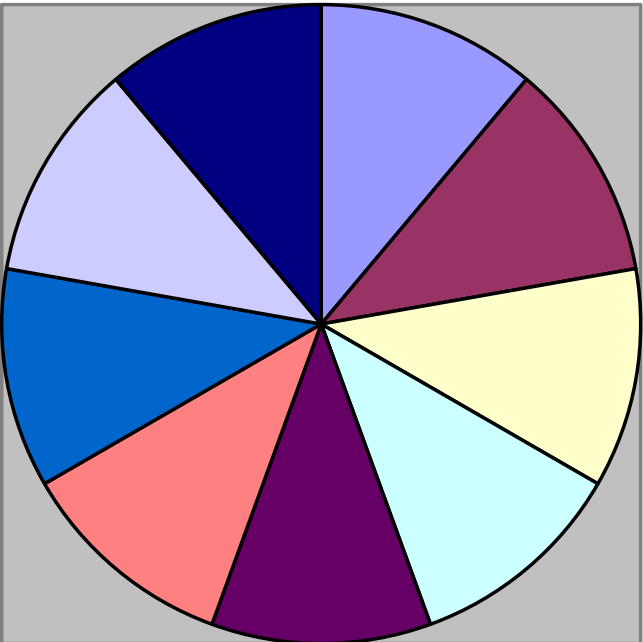

\section{$\square$ Development of drugs $\square$ Diagnostics of diseases \\ $\square$ Health planning and analysis Tracking of health}

Smart consultations

\section{Surgical diagnostics}

$\square$ Clinical data management $\square$ Custom diagnostics

\section{- Healthcare diagnostics}

Fig 2. AI application within the healthcare sector

Radiography has been the sector that is the most open to adopting new technologies. Machines were first employed in diagnostic practice for administration tasks such as image collection and preservation, but with the introduction of picture preservation and telecommunication networks, they have now become an essential part of the work setting. The application of CAD (computer-assisted diagnostics) in mammography screening is widely documented. Due to the positive parameter estimates, sensitivities, and precision, recent investigations have shown that CAD does not offer much diagnostic help. Furthermore, false-positive classifications may cause the radiologist to get distracted, leading in needless work-ups. In radiology, AI may be useful in not only classifying abnormal examinations but also detecting fast negative examinations in computerized tomographies, X-rays, and neuroimaging, particularly in high-volume environments and institutions with limited human input.

The University of Massachusetts created DXplain in 1986, which provides a listing of possible differences depending on the symptoms complexity and is also used as an instructional technique for clinical learners to fill in the spaces not covered in traditional books [12]. The University of Washington designed Germwatcher to identify and evaluate hospitalacquired illnesses. Individuals in the United Kingdom can use an internet based program called Babylon to speak with their physicians, examine for signs, obtain guidance, track their condition, and purchase testing kits. Aside from that, AI's scope has broadened to include medicinal applications. The use of artificial intelligence (AI) in therapeutic is gaining popularity.

Innovative Surgical's advanced robotic technology has transformed surgeries, particularly urology and gynecology procedures. The program's robot arms have a 3D vision and magnifying features that enable the surgeons to execute minute cuts by mimicking a surgeon's arm gestures with greater accuracy. From 2018, Buoy Wellbeing and the Boston Children's Clinic have been collaborating on a web-based AI program that offers parental advise on their sick child by responding to questions regarding drugs and when signs warrant a doctor consultation. The National Institutes of Health (NIH) developed the AiCure App, which uses smartphones webcams accessibility to check a client's medicine use and thereby minimize non adherence levels.

Fitbit, Apple, and other wellness monitors can detect heartbeat, exercise habits, and sleeping patterns, and some have even added ECG reconstructions to their offerings. All of these new advancements can notify the user to any changes and 
provide the physician with a greater understanding of the specific patient. The Netherlands employs artificial intelligence to analyze its health system, finding therapeutic errors and inconsistencies in order to reduce avoidable hospitalization [13]. Aside from existing innovations, several advancements are in different stages of exploration that will assist doctors in becoming general practitioners. IBM's Watson Healthcare is a good illustration of this, as it will be able to detect heart problems and tumor indications quickly. A system for Intelligence healthcare is being developed at Stanford University (PAC). Through the use of many detectors, PAC has developed a sophisticated senior well-being assistance system and sophisticated ICUs that will detect any behaviour change in older adults residing independently and ICU victims. Smart Handwashing Assistance and Health insurance Conversation Interfaces are two other initiatives that PAC is working on.

Depth detectors and computational vision technologies are being used to provide ideal hand washing for physicians and nurses' personnel, minimizing healthcare associated infections. Medical conversation initiatives examine how Siri, Google Now, S voice, and Cortana responds to queries from portable device users about mental wellbeing, antisocial behaviour, and physical wellbeing, enabling individuals to access treatment sooner. Molly is a computerized nurse who will give follow-up treatment to released individuals, enabling physicians to concentrate on more urgent problems. In the future, AI will play a significant role in medicine. As a result, it is critical to educate the next generation of medical students about the principles and applications of AI, as well as how to operate effectively in a workplace alongside robots for increased efficiency, while also developing soft skills such as empathy.

In the early 1970s, "the prospect that the computer as an intelligent device can reconfigure the current network of providers, dramatically change the responsibility of the health care professional, as well as tremendously affect the dynamics of clinical personnel enrollment and professional examinations short, the likelihood that health service by year 2000 will be substantially different from how it is presently" was discussed. Nations such as Finland, Germany, the United Kingdom, Israel, China, and the U.S. are all heavily investing in this area. The United States remains the "quantitative champion," with the largest list of enterprises with the highest market capitalisation and widest trials and research studies; China has the largest population increase in medical Digitalization and strenuous customer strategy (for instance -, Ping An Good Doctor); and Countries in europe have economic benefits in terms of scope of accumulated clinical information and the number of joint research studies in various issues of AI use in medical science, such as data security. As a result, there is no one "flagship" at this time, and the use of AI in healthcare is only getting started. When we get down to semantics (its abbreviation - AI), we immediately run into certain complications. One of the first general definitions sounded something like this: "Artificial Intelligence (AI) is the examinations of concepts that allow computers to do tasks that make humans seem clever... Artificial Intelligence's main objectives are to make machines more helpful and to comprehend the concepts that allow intelligence to exist." We now have some "legislative" definitions.

As a result, the European Commission's ethical AI guideline includes the following terminology: "Artificial intelligence (AI) frameworks are application (and possibly components) programs developed by humans that act in the real or virtual component by interpreting their surroundings through data capture, analysing the accumulated semi - structured and unstructured data, rationalization on the experience and understanding, or analysing data, derived from this documentation, and select the most suitable intervention(s) to consider taking to accomplish the performance target. Intelligent machines may adopt a numeric framework or utilize symbolic rules, and they can modify their behavior by evaluating how their past actions have impacted the environment. The United States' methodology, on the other hand, is based on the concept of "augmented intelligence," emphasizing the improved ability of the human medical decision making whenever combined with these computer techniques and systems." Both methods offer benefits and levels of reasoning, but the two most important factors to examine are: 1) that AI is currently construed in three ways: as a simplified instrument with no level of independence (such as an electronic aide or "spreadsheet"), as an institution with some independence but under human influence, and as an institution with broad independence, completely or partially replacing human behaviour, and we must acknowledge that first one could be taken into account AI at all during the present state of the science growth; 2) definition of AI We all (or at least for the most of us) use AI-enabled assistants, and the trend is increasing. We favor the term "artificial intelligence" over "augmented intelligence" since the latter, in our opinion, allows a lot of room for "human oversight" connotation, which will restrict the meaning of AI as it develops in the future.

Therefore, what is artificial intelligence (AI) in medicine? Simply put, medical AI. AI in healthcare profession now consists of 3 technical forms: hardware, software and hybrid types. Software solutions vary from simple fixation and document to artificial neural techniques that generalize data and suggest treatment choices based on their efficacy. The majority of AI in healthcare's software-based possibilities could be demonstrated in the following aspects: Artificially Intelligent Strategies in Healthcare; Big Data and Knowledge Extraction in Healthcare; Medical Optimization Techniques; Machine Learning-Based Pharmaceuticals; Clinical Signal as well as Image Postprocessing Hardware is a "universe of robots" that aids with medical care, surgery, recuperation, and smart prosthetics, among other things.

The blended form combines both aspects as parts of a larger structure. In reality, there are just handful "almost-proper" instances. Medical treatment is confronted with two contemporary issues that AI may help solve. The increase of "large datasets" - massive quantities of statistics from a variety of source materials (computerized clinical documentation (EMR, systematic and useful medical publications, clinical testing and with their findings, insurance data, pharmacist documents, knowledge contributed by sick people through the use of mobile phones, wearable technology, and so on) - and the requirement (and capacity) to classify and discover regular patterns to improve patient healthcare and diagnosis. These two issues have resulted in a single solution: the need for mechanization and help. It's strange, but the medical system, which is supposed to be receptive to new technology, has one of the lowest conceivable automation rates - just $15 \%$ of work times are expected to be automated by 2030 , but only $35 \%$ are theoretically automated at all. 
Scientific proof analysis adds to the pessimism, saying that "health has a poor track history for embracing actually cutting technology." What role might artificial intelligence play in this? Medicinal technology's main approach is to create linkages via trends depending on current information (datasets), and we can assume that the statistical technique existed at the heart of this procedure for a long period of time even before AI. However, utilizing three major scientific-statistical methods - the designed method, the databases technique, and the strategic decision method - AI might be much more successful in this area. They're all usable, but they're all better suitable for AI application in various ways. The "Diagrammatical technique" is based on the collection of known signs, which results in a historical record and a likely diagnosis by integrating signs into one image. The drawbacks of such a strategy are apparent. They are the need to enter a large amount of data on various symptoms, their features, combinations, related illnesses, and so on in order to get a reliable result. Furthermore, because of healthcare professional's intermediate function, such a strategy is restricted - the AI Algorithms can't "query" anything other than the data given by the clinical employee, and can't get any data about the specific patient - a 100 percent "machines" and predictable method.

It may, nevertheless, be helpful in some situations, such as recording emergency procedures for clinicians to follow, individual questioning, or providing medical recommendations in the chemical region. The "Data approach" is centered on the personality, personal self, and in analytical principles. When AI has to understand how to detect interconnections and trends, it employs a set of techniques that are intended to determine how illness or mixes of disorders, visual aspects, and other factors emerge. Such technologies are typically operational, for example, in the relevant problem of COVID-19 diagnoses based on coughed sounds. Although such information computing technologies have progressed, they can be used in all situations due to a number of concerns, including elevated expenses and duration expended gathering and manufacturing huge datasets; difficulties comparing old and new information; cultural variations in gathered information; the potential of clinical exclusions in certain kinds of infections; and a lack of specifics and incapability to replace the responsibility of the doctors. Certainly, such a technique may be accomplished in practice, but the breadth of such deployment should not be overstated.

The "strategic decision technique" is based on statistical methods for taking decisions under ambiguity, which include previous experiences, manifestation, probability, and consequences. Despite its simplicity, such a method has certain difficulties about getting acceptable estimations of probability and benefits for a specific study. Whereas methods like sensitive assessment may assist to identify which possible errors are insignificant, a limitation of sufficient information frequently induces false approximations of the issue, lowering trust in the study' conclusion. For example, such a method may lead to a scenario in which many signs are mistaken for a singular illness (when they are really a composite resulting to one), or vise - versa. Furthermore, the quantitative analytic depiction of the strategic decision procedure clearly varies from those of a genuine personal clinician, which may cause the patients to be confused. As a result, each method is appropriate, but not ubiquitous. Moreover, considering the fairly lengthy history of AI, we must acknowledge that it is just the beginning for the technologies in overall and its application in medical in specific. "While there are broad concerns about what is genuine in AI in medical presently," based to new study, "this analysis examined at 23 programs in use now and offers example reports of 14 programs that are currently being used. These examples show how AI may help individuals control their own treatment, as well as computerized symptoms detectors and e-triage AI systems, digital assistants that can do jobs in clinics, and a prosthetic pancreatic to assist diabetic individuals." According to predictions, there will be three major stages of AI in medical scalability:

1. A low technological application layer in which AI will help with administration chores that are repetitious. At this time, AI technologies will allow actual image AI application in ophthalmic and radiography, as well as reducing the associated burden (not the primary one) of clinical personnel at all stages.

2. AI-assisted residence healthcare stage, in which AI-assisted distant tracking and alarming visible guidance on the foundation of AI technologies will enable a shift in specialized health models towards distant surveillance and detecting visible help. Furthermore, breakthroughs will be made in AI's application in cancer care, cardiothoracic, and neuroscience, where it is currently demonstrating its first aspects of deployment with wider digital supply chain configurations (by machine learning, NLP, and accessibility) and organizational growth in conjunction with established innovations.

3. Medical studies and judgement support level, in which AI innovations will be integrated into clinical information systems and entrenched in every step of the care systems, from teaching and understanding the medical studies and diagnosis, as well as healthcare improvement and overall care assessment.

As a result, we are still a long way from widespread AI adoption in medical, and now is the time to consider what kinds of problems it may bring. Are we prepared to deal with them, and if not, what approach should we use to reduce the dangers? Moreover, the fundamental problem stems from the fact that we're talking about fundamental changes in medical, public health concerns, the clash among formal and informal concerns, values and ethical, innovation, and mankind. We have no plans to go extensively into every aspect of AI application in health, and it will be the focus of our future study. So, let's go to work on the research.

\section{DISCUSSION}

The deployment of machine-learning technologies and techniques, or intelligent systems, to replicate mental abilities in the assessment, display, and interpretation of complicated healthcare and health data is known as artificial intelligence in medicine. AI is the capacity of computational models to make educated guesses based only on data input. AI technology is distinguished from traditional healthcare solutions by its ability to take data, evaluate it, and offer a well-defined 
consequence to the end-user. AI does this via the application of computer engineering and deep learning. These algorithms can recognize patterns in social cognition and generate reasoning on their own. In order to generate useful insights and forecasts, deep learning systems must be trained using enormous amounts of data. There are two ways that AI systems vary from living beings: (1) Techniques are accurate: once a goal has been achieved, the machine learns primarily from the data input and could only fully comprehend what it has been conditioned to do; (2) Some deep learning methods are mechanical devices: they can anticipate with absolute accuracy but provide almost no intelligible elaboration of the reasoning behind their decision making, aside from the documentation as well as category of automated system used.

The primary purpose of health-related Artificial intelligence systems is to look at the correlations between prevention and therapeutic techniques and clinical results. Information systems are being employed in diagnoses, treatment protocols, drug research, tailored medicine, and patients conditions of patients, among other fields. In addition to illness prediction and treatment, Computer algorithms may be used to examine massive volumes of data from electronic health records. AI algorithms have been created by medical institutes like the Mayo Clinic, Memorial Sloan Kettering Medical Group, and the British National Health System. AI algorithms for healthcare have been created by major technology firms such as IBM and Google. Healthcare facilities are also turning to AI technologies to help them with cost-cutting, improved patient, and staffing and labor requirements. The US government is now spending millions of dollars on AI research in the healthcare sector. Companies are creating solutions to assist healthcare executives in improving utilization, minimizing patient embarkation, minimizing length of stay, and managing size of the workforce in order to enhance business operations. Because AI is still relatively new in medicine, there are a slew of new moral problems, including data privacy, employment displacement, and representational biases.

Dendral, a problem-solving software or intelligent systems developed in the late 1960s and early 1970s, was the first of its kind. While it was created for organic chemistry implementations, it served as the foundation for MYCIN, one of the first application areas of AI in healthcare. MYCIN and other technologies like CASNET, on the other hand, haven't found widespread adoption among practitioners. The microprocessor and new degrees of network connection proliferated over the 1980s and 1990s. During this period, researchers and engineers realized that AI technologies in medicine needed to be developed to work around the lack of accurate data and rely on doctors' knowledge. Intelligent computer systems in healthcare have used approaches such as fuzzy sets, Neural nets, and convolutional neural networks.

The expansion of healthcare-related AI has been facilitated by medical and technology breakthroughs over the last half-century, including:

- Faster data collecting and processing are the consequence of increased computer power.

- The increase in the number of databases for genetic research

- Electronic health records are becoming widely implemented.

- Machines can now mimic human sensory experiences because to advances in language generation and analytics tools.

- Robot-assisted surgery has become more precise.

- In rare illnesses, advancements in deep learning algorithms and data logs

Artificial intelligence (AI) is making great progress in the medicare program, and it will have a substantial impact on all areas of primary care. Because of AI-enabled computing applications, basic healthcare professionals will be able to correctly identify people who need further attention and provide individualized regimens for each. Artificial intelligence might be used by basic healthcare professionals to keep track of notes, analyze patient interactions, and enter data directly into EHR systems. These applications will collect and assess patient data before offering them to general practitioners with data about their medical needs. According to a 2016 study, physicians invest 27 percent of their workday in clinical settings engaging in face-to-face interactions with their patients, and $49.2 \%$ on electronic patient records and paperwork. 52.9 percent of practitioners' time was spent in the examination room with patients working on EHR and associated duties [14]. Finally, doctors who utilized documented services and support such as transcription help or clinical transcribe programs spent more continuous encounter contact with individuals than doctors who did not. Furthermore, increasing AI use in medical improves efficiency, accuracy, and effectiveness while reducing human work and freeing up time for general practitioners.

Medical studies take decades and require a billion dollars to find and improve pharmacological medicines for a particular illness. To provide an alternative, AI was recently utilized to evaluate current medicines that might be used to combat the growing Ebola disease threat, a task that could have lasted for decades to complete otherwise. We would not have been able to embrace the new idea of "precise treatment" with the assistance of AI. In certain research, AI Algorithms have been shown to surpass physicians in accurately identifying worrisome skin conditions. This is due to the fact that AI technologies may learn more from subsequent instances and can be subjected to numerous examples in moments, considerably beyond the amount of cases a physician could assess in a single human lifetime. In instances where specialists frequently differ, such like diagnosing respiratory TB on chests radiography, Intelligence strategic decision methods are employed. There are as many doubters as supporters in this new age of AI-assisted practicing (see Table 1). Many physicians in training and working physicians are worried that the increasing use of technologies has decreased the variety of employment possibilities. Computers may be enabled to interpret people's behavior logically and rationally, but critical reflection, communication and interpersonal abilities, emotional maturity, and inventiveness are human qualities that cannot be developed by machinery. 
Table 1. Advantages and disadvantages of AI-assisted practicing

\begin{tabular}{ll}
\hline Advantages & Disadvantage \\
\hline $\begin{array}{l}\text { Saves money, enhances monitoring } \\
\text { Decreased workloads, enhances patient face time, } \\
\text { enhances the time on crucial patient cases }\end{array}$ & $\begin{array}{l}\text { Lob loss } \\
\text { emotional intelligence }\end{array}$ \\
\hline $\begin{array}{l}\text { Effectiveness, precision and accuracy } \\
\text { emothe and touch, availability of }\end{array}$ \\
\hline
\end{tabular}

The Digitized Mammogram DREAM Competition was held in 2016, with the aim of establishing a Machine learning methodology via the evaluation of 640,000 mammogram images. The top results were 0.8 selectivity, 0.8 sensitivities, and 0.9 square underneath the receiver - operating characteristic curve, approximately equal to the lowest $10 \%$ of physicians. Finally, although AI has promise, it is doubtful that it will ever completely replace physicians.

\section{CONCLUSION}

Finally, it is critical that primary care doctors become well-versed on forthcoming AI advancements and the new uncharted area that the field of medicine is entering. The aim should be to achieve a delicate, cooperatively advantageous equilibrium between the efficient use of robotics and artificial intelligence (AI) and the human capabilities and judgment of qualified primary care doctors. This is necessary since the possibility of AI fully increasing automation in the branch of healthcare is a worry that might otherwise limit the advantages that can be obtained. As previously stated, if we clarify them in terms of healthcare, we are executing the method of obtaining the judgment, which would be usually either 1) indescribable at all, since some of the phases are "assumed" or "missed"; or 2) comprehensible, but the interpretation is structurally warranted but makes even less sense from the a clinical perspective. That is why the phrase "data recorder" is often used to describe the decision-making mechanism in advanced technologies, since the initial algorithm may be modified as a result of a large quantity of data being examined or as data transforms over time. This may be beneficial when looking at picture identification and early detection of diseases like skin cancer, detecting problems in newborns based on facial characteristics, suggesting off-label usage of current medicines, and so on. However, medical research and therapy must be founded on a reasonable degree of confidence, thus ensuring efficacy and safety while not disrupting AI technological innovation and usage would be a difficult task.

\section{References}

[1]. A. Sana, S. Razzaq and J. Ferzund, "Automated Diagnosis and Cause Analysis of Cesarean Section Using Machine Learning Techniques", International Journal of Machine Learning and Computing, pp. 677-680, 2012. Doi : 10.7763/ijmlc.2012.v2.213.

[2]. R. Moesinger, C. Hall and J. Reese, "W1655 Colorectal Surgical Specimen Lymph Node Harvest: Improvement of Lymph Node Yield with a Physicians Assistant", Gastroenterology, vol. 134, no. 4, p. A-900, 2008. Doi : 10.1016/s0016-5085(08)64229-8.

[3]. A. Haldorai and A. Ramu, "Canonical Correlation Analysis Based Hyper Basis Feedforward Neural Network Classification for Urban Sustainability," Neural Processing Letters, Aug. 2020. doi:10.1007/s11063-020-10327-3

[4]. S. Reardon, "AI algorithms to prevent suicide gain traction", Nature, 2017. Doi : 10.1038/d41586-017-08307-0.

[5]. A. Hadioui, N. El Faddouli, Y. Benjelloun Touimi and S. Bennani, "Machine Learning Based On Big Data Extraction of Massive Educational Knowledge", International Journal of Emerging Technologies in Learning (iJET), vol. 12, no. 11, p. 151, 2017. Doi : 10.3991/ijet.v12i11.7460.

[6]. "Interview with Matthew Lungren, MD, MPH - Stanford Medicine Big Data | Center for Artificial Intelligence in Medicine \& Imaging", Aimi.stanford.edu, 2021. [Online]. Doi : https://aimi.stanford.edu/news/interview-matthew-lungren-md-mph-stanford-medicine-big-data. [Accessed: 23- Oct- 2021].

[7]. A. Esteva and E. Topol, "Can skin cancer diagnosis be transformed by AI?", The Lancet, vol. 394, no. 10211, p. 1795, 2019. Doi : $10.1016 / \mathrm{s} 0140-6736(19) 32726-6$.

[8]. "Hey Google. Is This Lung Cancer?", GEN - Genetic Engineering and Biotechnology News, 2021. [Online]. Doi : https://www.genengnews.com/insights/hey-google-is-this-lung-cancer/. [Accessed: 23- Oct- 2021].

[9]. D. Nelson, "Artificial Intelligence Being Trained To Combat DeepFakes", Science Trends, 2018. Doi : 10.31988/scitrends.41280.

[10]. "Los compradores de Instacart planean una serie de acciones en protesta a las prácticas salariales de la empresa HYPERNOIR", HYPERNOIR, 2021. [Online]. Doi : https://hypernoir.com/en/mendelics-debuts-dna-test-brazil-pandoras-genome/. [Accessed: 23- Oct- 2021].

[11]. P. Boddington, P. Millican and M. Wooldridge, "Minds and Machines Special Issue: Ethics and Artificial Intelligence", Minds and Machines, vol. 27, no. 4, pp. 569-574, 2017. Doi : 10.1007/s11023-017-9449-y.

[12]. D. Devikanniga, A. Ramu, and A. Haldorai, "Efficient Diagnosis of Liver Disease using Support Vector Machine Optimized with Crows Search Algorithm," EAI Endorsed Transactions on Energy Web, p. 164177, Jul. 2018. doi:10.4108/eai.13-7-2018.164177

[13]. S. Roffel, "Introducing article numbering to Artificial Intelligence", Artificial Intelligence, vol. 278, p. 103210, 2020. Doi : 10.1016/s00043702(19)30204-8.

[14]. J. EW and H. Grove D, "Physician Opinions about EHR Use by EHR Experience and by Whether the Practice had optimized its EHR Use", Journal of Health \& Medical Informatics, vol. 7, no. 4, 2016. Doi : 10.4172/2157-7420.1000240. 